\title{
Newborn hearing screening in a South African private health care hospital
}

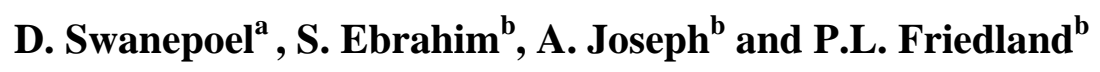 \\ ${ }^{\mathrm{a}}$ Department of Communication Pathology, University of Pretoria, Pretoria, Gauteng \\ 0002, South Africa \\ ${ }^{\mathrm{b}}$ Donald Gordon Medical Centre, University of the Witwatersrand, South Africa
}

\section{Summary}

\section{Objective}

Early Hearing Detection and Intervention (EHDI) programs are being established as part of the public health systems in increasing numbers of countries. In developing countries, however, little progress has been made towards implementing NHS programs and South Africa's public and private health care sectors is no exception. The current study presents the first report on a hospital-based UNHS program conducted in the South African private health care sector to provide preliminary results towards advocating for and guiding future programs.

\section{Methods}

A retrospective study of a UNHS program at a private hospital in urban Gauteng, South Africa over a 4 year period of time was performed. Screening was conducted with Transient Evoked Otoacoustic Emissions (TEOAE) with a rescreen recommended within 6 weeks if referred. Diagnostic audiological assessments were performed on those infants referring the rescreen. The discharge screening costs were subsidized through the hospital birthing package for the first 22 months of the program. 


\section{Results}

Six thousand two hundred and forty-one newborns were screened from 13,799 hospital births during the first 4 years. Ninety-four percent of these infants were from the well-baby nurseries. During the initial 22 months, whilst the service was subsidized as part of the hospital birthing package, coverage of $75 \%$ was attained compared to $20 \%$ during the subsequent 26 months. The overall referral rate for the screening program across the 4 years was $11.1 \%$ but referral rates decreased by between 2 and 4\% for each year of program existence with a 5\% rate in year 4 . Only $32 \%$ of the rescreens were completed at the hospital and no data was available for the remaining infants because parents were provided a choice of follow up centers.

Referral for a diagnostic assessment after the rescreens at the hospital was predictive of sensorineural hearing loss in one-third of cases and the estimated prevalence was 3 in every 1000.

\section{Conclusions}

Screening coverage in the current study was not adequately high and can be attributed to insufficient parental knowledge to make an informed decision. Improvements in program efficiency over time also suggest that pilot programs must be monitored over sufficiently long periods of time to allow observations of optimal efficiency. Initial referral rates and prevalence data indicate a large hearing loss burden and the capacity to implement increasingly efficient programs in South Africa.

\section{Article Outline}

1. Introduction

2. Materials and methods

3. Results

4. Discussion

5. Conclusion

References 


\section{Introduction}

Early identification of hearing loss through newborn hearing screening (NHS) programs has proven to be the most effective way of identifying affected children early enough to ensure optimal outcomes [1]. NHS yields dramatic benefits, since infants whose hearing loss is identified before 6 months of age have significantly better language abilities compared to those whose hearing loss were identified later [2], [3] and [4]. Intervention before the age of 6 months enables infants to develop and maintain language skills on par with their cognitive development and in the average range of age-appropriate language skills. This is in stark contrast with the below average language skills demonstrated by infants identified after 6 months of age [2]. The high prevalence of childhood hearing loss and its amenability to early intervention emphasises the importance of screening for the condition. It is not surprising therefore that Universal Newborn Hearing Screening (UNHS) has become a powerful professional and technological movement with widespread influence within developed countries such as the USA and UK [5]. Early Hearing Detection and Intervention (EHDI) programs are established as part of the public health system in both of these countries and 95\% of all newborns in the United States are being screened before discharge. Many other countries are following the examples set by these countries and Poland for example now screens 99\% of all newborns before they leave the hospital [6]. It is clear therefore that UNHS is becoming part of standard medical care for neonates [7].

In a country like South Africa, however, the vast majority of children have no prospect of having their hearing screened despite a reasonably established health care infrastructure compared to other developing countries [12], [13] and [14]. A limited number of small NHS programs are spread across the public and private health care sectors [13]. Unfortunately, there are no surveys documenting the current status of early identification of hearing loss in South Africa. Existing programs are often insufficiently managed and supported within hospitals without systematic data capturing. Apart from a recent report on infant hearing screening at immunization clinics there are no other published reports on NHS in South Africa [10]. This is despite an increased risk amongst South African infants for hearing loss due to environmental risks, especially the burden of infectious diseases such as HIV/AIDS [15]. 
The South African health care sector is characterized by widespread inequality due to an inherited system from the apartheid era that was originally intended to primarily benefit the white minority. The majority of South Africans, comprising approximately 80-85\% of the population, are currently served by a public healthcare sector, which only utilizes 39\% of the countries' total health care expenditure. This is in stark contrast to a wealthy minority, comprising 15-20\% of the population, which make use of private health care services that constitute $61 \%$ of the total health expenditure [16]. Private health care services in South Africa are therefore exclusively reserved for those who can afford to belong to a private medical insurance scheme or who can pay for services out of pocket. Despite these large disparities, however, the public health care sector has succeeded in increasing access to the majority of the population as is evident in the high rates of antenatal care and assisted deliveries compared to other countries in Sub-Saharan Africa [17].

Despite more advanced health care in private hospitals few newborn hearing screening programs are being implemented [13]. Recent reports from South Africa has demonstrated initial developments towards NHS in the public health care sector with no reports on the status of screening in the private sector. The dearth of reports evidences the fact that few hearing screening programs are being conducted in both public and private health care settings [13]. Since it is clear that funds are much more accessible in the private health care sector it is worrisome that even in these settings newborn hearing screening is not part of standard neonatal medical care. Various factors including a lack of awareness amongst gatekeeper health care professionals and parents regarding the prevalence of infant hearing loss and the dramatic benefits of early intervention, may be attributing to the current state of screening in South Africa.

Pilot studies serve as an essential means to advocate the importance of early identification of hearing loss followed by subsequent intervention. Research evidence from such studies provides epidemiological data in support of screening programs and guides the development of contextually relevant protocols. In a country with no true prevalence data for hearing loss the current study presents the first report on a hospital-based UNHS program conducted in the South African private health care sector to provide preliminary results towards advocating for and guiding future UNHS programs. 


\section{Materials and methods}

The institutional review board of the hospital in which the screening program was conducted approved this project before any data collection commenced.

Results of a UNHS program at a private hospital in urban Gauteng, South Africa, over a 4-year period of time are presented. The program was initiated in September 2001 and results are reported until August 2005. Initially, the hospital administration funded the screening as part of the hospital package offered from September 2001 until June 2003 (22 months). From July 2003 until August 2005 (26 months) the hearing screening costs were not subsidized through the hospital birthing package and parents had to pay for the service out-of-pocket or through a private medical scheme. Screening costs were charged at the National Health Reference price level (approximately US\$25). In both the subsidized and non-subsidized screening periods informed consent was required by parents before a baby could be screened since it is not currently viewed as a high priority health procedure.

The screening was conducted by two qualified audiologists using Transient Evoked Otoacoustic Emissions (TEOAE). The automated Biologic AuDX TEOAE equipment in the study presented nonlinear clicks at $84 \mathrm{~dB}$ peak equivalent SPL. The stimulus parameters for the Biologic AuDX automatically determines the stimulus level, stopping rules and pass/fail result based on preset criteria. A 70\% reproducibility and a 6-dB signal-to-noise ratio at 2, 3 and $4 \mathrm{kHz}$ was set as the pass criteria. Both neonatal intensive care unit (NICU) and well-baby nursery infants were screened before discharge in a room with acceptably low noise levels. Screening was conducted every day of the week except on Sundays. The minimum hospital stay for mothers after birthing was $24 \mathrm{~h}$ which allowed enough time to screen almost all babies. A small percentage were, however, missed before discharge and were later followed up. The target disorder was bilateral and unilateral sensorineural hearing loss of $35 \mathrm{~dB}$ and greater in the speech frequencies. The OAE screening was often repeated a second or third time if the infant did not pass for both ears on the first attempt. A TEOAE rescreen with a similar criterion was scheduled within 6 weeks at the hospital or at another audiologist of the parents' choice. Care was taken to give parents a choice of practicing audiologists they could consult for the rescreen. If an infant referred the TEOAE again on the rescreen at the hospital, he/she was referred to the Pediatrician or Ear, Nose and Throat Specialist and then to the diagnostic audiological 
clinic at the hospital. Follow-up visits were not integrated with routine perinatal follow-up since in the private sector parents attend these sessions with the pediatrician of their choice. Diagnostic assessments at the hospital included acoustic immittance measurements, $\mathrm{OAE}$, and the auditory brainstem response (ABR). Intervention services for these infants were usually coordinated by audiologists who conduct the hearing aid fitting and monitoring. Private early intervention services, such as speechlanguage therapy, services are available to these children in addition to a number of private schools for children with hearing loss.

Screening, follow-up and diagnostic assessment results were documented electronically but no risk factors or other patient information such as race or gender were documented in the electronic database. Collected data was captured electronically and subsequently analyzed over the 4-year period of time to ascertain the program performance.

\section{Results}

Of 13,799 hospital births during the first 4 years of the programs' existence only 6241 newborns were screened (45\%). The vast majority of these infants (94\%) were from the well baby nursery with only $6 \%$ from the NICU. The coverage of the screening program is illustrated in Fig. 1. During the initial 22 months, whilst the service was subsidized as part of the hospital birthing package, coverage of $75 \%$ was attained compared to $20 \%$ coverage during the subsequent 26 months when parents were directly responsible for payment.

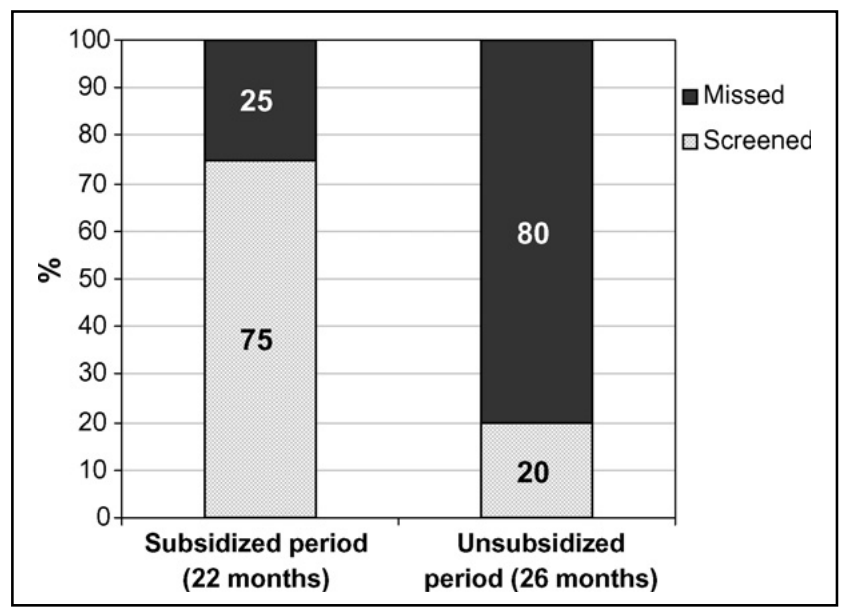

Fig. 1. Screening coverage of hospital births. 
An overall referral rate for the screening program across the 4 years was $11.1 \%$. The NICU referral rate was $6.7 \%$ compared to $11.4 \%$ in the well-baby nursery. Fig. 2 illustrated the steady decrease in overall referral rate from the first to fourth year of program existence. The referral rate decreased by between 2 and $4 \%$ for each year the program existed.

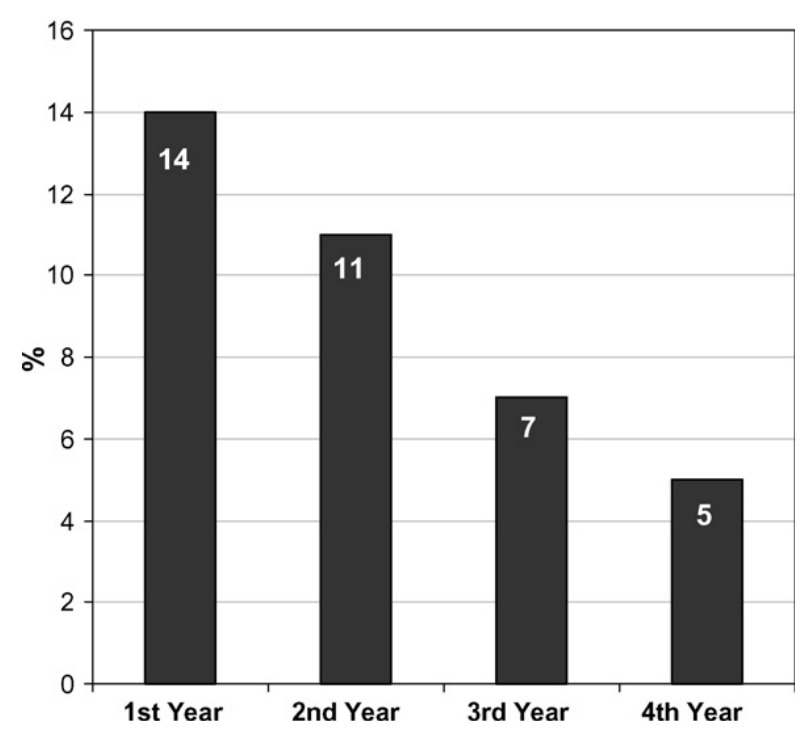

Fig. 2. Referral rate for successive years of program existence.

Rescreens were completed at the hospital in 32\% (219/694) of cases referring the discharge screen. A small percentage (9\%) of infants who received a rescreen was recommended for diagnostic testing. All 19 infants referred for diagnostic testing returned and 32\% of these cases presented with sensorineural hearing loss. These cases represent a 1/1000 prevalence of hearing loss but do not account for two-thirds of the infants referred for rescreens but who did not return for them at the hospital. Based on the prevalence of hearing loss amongst the 32\% of referred cases that returned to the hospital a prevalence of 3/1000 infants with sensorineural hearing losses can be estimated for this population.

The six identified cases with sensorineural hearing loss included four bilateral hearing losses ranging from moderate-severe to profound in degree and two cases of unilateral loss. 


\section{Discussion}

Research evidence of early hearing detection programs in developing countries, especially in Sub-Saharan Africa, is extremely limited. Initiating and reporting on pilot studies is an important step towards providing contextual evidence for the burden of childhood hearing loss and the efficacy of screening programs in these countries [18]. Research evidence must guide the expansion and widespread implementation of services to meet the existing health care need. Results from the current study provide the first indication of screening protocol efficiency and hearing loss prevalence in the South African private health care sector. Although not representative of the majority of the population who only have access to public health care facilities the current results provide important baseline information in a context where none previously existed.

A basic measure of screening efficiency is the coverage rate of the targeted population. During the initial two years of the study when the screening costs were included in the birthing package of the hospital the coverage rate was reasonably high at $75 \%$ of all births. This does not however meet the recommended coverage by the Joint Committee of Infant Hearing (JCIH) of 95\% within 6 months of program initiation [19]. The reduced coverage rate can be attributed to two main factors previously also reported for other studies [20]. Firstly, a number of parents did not provide consent for the screening. This may be avoided to a large extent by providing parents with necessary information regarding the importance of early identification before providing the hearing screen, preferably during antenatal classes or scheduled physician appointments prior to the birth [18]. Surveys on knowledge and perspectives on infant hearing loss and early intervention amongst South African parents are still lacking and should be pursued as a matter of urgency. Secondly, in a small minority of cases parents were discharged from hospital before the screening could be conducted. This emphasizes the need for quality monitoring and control of screening programs against the benchmarked indicators [21]. Hospital personnel should be very much aware of the screening protocol to ensure that parents receive the screen before discharge and the data must be carefully captured and managed to provide regular feedback on program efficiency [21]. The screening coverage indicated a dramatic drop of 55\% after the service was not included in the hospital birthing package after the first 22 months. The screening 
service then became an optional "extra” for which parents were required to pay. In this condition only one-fifth of parents consented to have their newborn's hearing screened. This indicates that parents did not regard hearing screening worth the financial expense and suggest that they are unaware of the importance of early identification of hearing loss. This once again emphasizes the important need to assist parents by providing appropriate and timely information, preferably prior to the birth, allowing them to make an informed choice [22]. In addition to facilitating informed choice and applying quality control measures to monitor screen coverage, results suggest that structuring NHS as part of the birthing package in private health care hospitals may result in optimal coverage.

Another important measure of program efficiency lies in the referral rate of the screening program. The overall discharge referral rate of $11 \%$ for an OAE screening program targeting bilateral and unilateral sensorineural hearing loss is within the same range as similar reports in developed and developing countries [18] and [23]. The average NICU discharge referral rate (6.7\%) was less than the well-baby nursery discharge refer rate (11.4\%). This is probably attributed to the fact that newborns in the well-baby nursery were most often discharged within $24 \mathrm{~h}$ of birth resulting in earlier screening when OAE referrals are more common due to the presence of vernix or middle ear effusion [24]. Employing a combined OAE and AABR or only AABR screening protocol would have resulted in lower discharge referral rates but the screening costs would be higher [25]. A further aspect to consider is that OAE screening protocols will not identify neural hearing losses and therefore compromises its efficiency to identify all infants presenting with hearing loss. It is these trade-offs between the costs and efficiency of the various screening technologies and protocols that require careful consideration within the context of resources available to a health care system [26].

A positive trend was observed in the referral rate over time. Consistent improvements, as illustrated in Fig. 1, demonstrate that the program efficiency, in terms of referrals, improved as the program matured. The discharge referral rate in the 4th year of the programs' existence was only $5 \%$, which is very close to the recommended $4 \%$ referral to diagnostic service set by the JCIH [19]. Previous reports have shown similar findings [27]. Consideration of the progressive nature of program efficiency should therefore be taken into consideration when using pilot study data in developing countries [10]. Time is often required to establish the best protocols and to allow for 
necessary capacity building to reach the specified benchmarks for accurate identification of all infants with hearing loss.

The infants who referred the discharge screen were scheduled for a rescreen within 6 weeks. Because parents were provided with a choice of whether to return for the rescreen at the hospital or at an audiologist of their choice, accurate follow-up return rates could not be established. Only $32 \%$ of the rescreens were completed at the hospital and no data was available for the remaining infants. This emphasizes the importance of tracking infants scheduled for referrals who are at an increased risk of hearing loss. Programs must implement data management and tracking systems that will ensure infants are followed-up and the data documented. This is essential for accountable service provision to families and also to supply comprehensive data on program efficiency and effectiveness for quality control [21]. Only 19 of the 219 returning for the rescreen where finally referred for diagnostic assessment. This suggests that as much as $91 \%$ of the discharge referrals may have been due to an obstruction in the outer ear canal and/or middle ear or due to poor probe placement, which prevented measuring robust OAEs [24] and [28].

Referral for a diagnostic assessment after the rescreen was predictive of sensorineural hearing loss in one-third of the cases. Four infants presented with bilateral and two with unilateral hearing loss. The proportion of unilateral to bilateral losses identified in the current study is similar to those previously reported from developing countries approximating one in every three to four [29] and [30]. The estimated prevalence of sensorineural hearing loss extrapolated from the number of infants returning for the rescreen and who were assessed at the hospital amounts to 3 in every 1000. This number corresponds to reported figures from developed countries [31] and [32]. It is important to note, however, that all infants with a neural hearing loss (auditory neuropathy) would have been missed by the current screening program because it relied on OAEs, which assesses pre-neural functioning. The prevalence of sensory and neural hearing losses would therefore exceed the current estimated number of 3 in every 1000. Late-onset and progressive hearing losses will also significantly increase this prevalence figure over time [33] and [34]. 


\section{Conclusion}

There has been a growing interest and advocacy recently for creating equal opportunities for infants with hearing loss in developing countries through early detection and intervention programs [8], [18] and [35]. This has also become evident in South Africa where a growing interest is reflected in the dissemination of a Hearing Screening Position Statement by the Health Professions Council of South Africa [10], [12] and [36]. To continue this momentum however, contextual research must be generated to assess the burden of hearing loss and to guide the development of these services. The current study provides the first initial hospital based report in the private health care sector with preliminary data to guide future programs and investigations. A lack of parental awareness regarding the importance of early identification of hearing loss in the current study undermined the screening coverage especially once parents were directly responsible for screening costs. Informed consent must be facilitated by increasing parental awareness regarding infant hearing loss and the need for early intervention. Furthermore, the results suggest that pilot studies must be implemented over sufficiently long periods of time to allow for observations of optimal efficiency. To ensure maximal impact these programs must also employ stringent data management and patient follow-up systems to generate sufficiently accurate and detailed data to successfully advance the case for early hearing detection and intervention in private and public health care in South Africa.

\section{References}

[1] C. Yoshinaga-Itano, Levels of evidence: universal newborn hearing screening (UNHS) and early hearing detection and intervention systems (EHDI), J. Commun. Disord. 37 (2004), pp. 451-465.

[2] C. Yoshinaga-Itano, A. Sedey, D. Coulter and A. Mehl, Language of early- and later-identified children with hearing loss, Pediatrics 102 (1998), pp. 1161-1171.

[3] R. Calderon and S. Naidu, Further support of the benefits of early identification and intervention with children with hearing loss, Volta Rev. 100 (5) (2000), pp. 5384.

[4] M.P. Moeller, Early intervention and language development in children who are deaf and hard of hearing, Pediatrics 106 (3) (2000), pp. 1-9. 
[5] J.W. Hall III, Infant hearing impairment and universal hearing screening, $J$. Perinatol. 20 (2000), pp. S112-S120.

[6] C.C. Morton and W.E. Nance, Newborn hearing screening-a silent revolution, $N$. Engl. J. Med. 354 (2006), pp. 2151-2164.

[7] K.R. White, The current status of EHDI programs in the United States, Ment. Retard. Dev. Disabil. Res. Rev. 9 (2003), pp. 79-88.

[8] B.O. Olusanya, L.M. Luxon and S.L. Wirz, Benefits and challenges of newborn hearing screening for developing countries, Int. J. Pediatr. Otorhinolaryngol. 68 (2004), pp. 287-305.

[10] D. Swanepoel, R. Hugo and B. Louw, Infant hearing screening at immunization clinics in South Africa, Int. J. Pediatr. Otorhinolaryngol. 70 (2006), pp. 1241-1249.

[12] D. Swanepoel, S. Delport and J.G. Swart, Universal Newborn Hearing Screening in South Africa: a first world dream?, S. Afr. Med. J. 94 (8) (2004), pp. 634-635.

[13] D. Swanepoel, Audiology in South Africa, Int. J. Audiol. 45 (2006), pp. 262-266.

[14] World Health Organisation 2006, World Health Report, Geneva, 2006.

[15] D. Swanepoel, R. Hugo and B. Louw, Disabling infant hearing loss in a developing South African community: the risks, J. Disabil. Int. Dev. 2 (2005), pp. 7582.

[16] ANC Today, Responding better to the health care needs of communities, 2005:5(43), http://0-

www.anc.org.za.innopac.up.ac.za:80/ancdocs/anctoday/2005/at43.htm\#art1.

[17] S.R. Benatar, Health care reform and the crisis of HIV and AIDS in South Africa, N. Engl. J. Med. 351 (2004), pp. 81-92.

[18] B. Olusanya, D. Swanepoel, H. Castillo, M.J. Chap-chap, H. Habib and S.Z. Mukari et al., Progress towards early detection services for infants with hearing loss in developing countries, BMC Health Serv. Res. 7 (14) (2007).

[19] Joint Committee on Infant Hearing, Year 2000 position statement: principles and guidelines for early hearing detection and intervention programs, Am. J. Audio 9 (2000), pp. 9-29.

[20] C.R. Kennedy, L. Kimm, D. Cafarelli-Dees, M.J. Campbell and A.R.D. Thornton, Controlled trial of universal neonatal screening for identification of permanent childhood hearing impairment, Lancet 352 (1998), pp. 1957-1964. 
[21] T. Finitzo and S. Grosse, Quality monitoring for early hearing detection and intervention programs to optimize performance, Ment. Retard. Dev. Disabil. Res. Rev. 9 (2003), pp. 73-78.

[22] B.O. Olusanya, L.M. Luxon and S.L. Wirz, Infant hearing screening: route to informed choice, Arch. Dis. Child 89 (2004), pp. 1039-1040.

[23] S.J. Norton, M.P. Gorga, J.E. Widen, R.C. Folsom, Y.S. Sinninger and B. ConeWesson et al., Identification of neonatal hearing impairment: evaluation of transient evoked otoacoustic emission, distortion product otoacoustic emission, and auditory brainstem response test performance, Ear Hear 21 (5) (2000), pp. 508-528.

[24] R.T. Boone, C.M. Bower and P.F. Martin, Failed newborn hearing screens as presentation for otitis media with effusion in the newborn population, Int. J. Pediatr. Otorhinolaryngol. 69 (2005), pp. 393-397.

[25] B.R. Vohr, W. Oh, E.J. Stewart, J.D. Bentkover, S. Gabbard and J. Lemons et al., Comparison of costs and referral rates of 3 universal newborn hearing screening protocols, J. Pediatr. 139 (2) (2001), pp. 238-244.

[26] D. Swanepoel, B. Louw, R. Hugo, A novel service delivery model for infant hearing screening in developing countries, Int. J. Audiol., in press.

[27] A. Mehl and V. Thomson, The Colorado newborn hearing screening project, 1992-1999: on the threshold of effective population-based universal newborn hearing screening, Pediatrics 109 (1) (2002), pp. 1-8.

[28] A.R.D. Thornton, L. Kimm, C.R. Kennedy and D. Cafarelli-Dees, External- and middle-ear factors affecting evoked otoacoustic emissions in neonates, Br. J. Audiol. 27 (1993), pp. 319-327.

[29] P.E. Brookhouser, D.W. Worthington and W.J. Kelly, Unilateral hearing loss in children, Laryngoscope 101 (1991), pp. 1264-1272.

[30] P.M. Watkin, M. Baldwin and S. Laoide, Parental suspicion and identification of hearing impairment, Arch. Dis. Child 65 (1990), pp. 846-850.

[31] A. Mehl and V. Thomson, Newborn hearing screening: the great omission, Pediatrics 101 (1) (1998), pp. 1-6e.

[32] T. Finitzo, K. Albright and J. O’Neal, The newborn with hearing loss: detection in the nursery, Pediatrics 102 (1998), pp. 1452-1460.

[33] A. Davis, J. Bamford, I. Wilson, T. Ramkalawan, M. Forshaw and S. Wright, A critical review of the role of neonatal hearing screening in the detection of congenital hearing impairment, Health Technol. Assess. 1 (10) (1997), pp. 1-177. 
[34] C.M.T. Robertson, J.M. Tyebkhan, M.E. Hagler, P.Y. Cheung, A. Peliowsky and P.C. Etches, Late-onset, progressive sensorineural hearing loss after severe neonatal respiratory failure, Otol. Neurotol. 23 (2002), pp. 353-356.

[35] B. Olusanya, B. McPherson, D. Swanepoel, R. Shrivastav and M. Chap-chap, Globalization of infant hearing screening: the next challenge before JCIH?, J. Am. Acad. Audiol. 17 (2006), pp. 293-295.

[36] D. Swanepoel, S. Delport and J.G. Swart, Equal opportunities for children with hearing loss by means of early identification, S. Afr. Fam. Pract. 49 (1) (2007), p. 3.

Corresponding author. Tel.: +27 12 4202304; fax: +27 124203517. 